\title{
DIFERENCIAS DE PERFECCIONISMO EN FUNCIÓN DEL GRADO DE RECHAZO ESCOLAR POR EVITAR LA AFECTIVIDAD NEGATIVA QUE PROVOCAN LOS ESTÍMULOS ESCOLARES
}

\author{
Mํa Pilar Aparicio Flores \\ Universidad de Alicante \\ pilar.aparicio@ua.es \\ Virginia Narcisa Ortega Sandoval \\ Universidad Central del Ecuador \\ Aitana Fernández Sogorb \\ Universidad de Alicante \\ José Manuel García Fernández \\ Universidad de Alicante
}

Fecha de Recepción: 27 Mayo 2019

Fecha de Admisión: 25 Septiembre 2019

\section{RESUMEN}

El perfeccionismo es entendido como aquella tendencia a establecer elevados estándares de desempeño, acompañado de una evaluación excesivamente crítica de los mismos y una gran preocupación por cometer errores, cuestión por lo que se relaciona con diversos problemas patológicos. A su vez, uno de los factores de rechazo escolar, evalúa el rechazo de estímulos que provocan la afectividad negativa, referente a la ansiedad que provoca la obligación de asistir y permanecer en la escuela. Es decir, aquellos sentimientos negativos que puede provocar en algunos sujetos. El objetivo planteado en el siguiente estudio fue analizar si existen diferencias estadísticamente significativas de perfeccionismo en adolescentes con altas y bajas puntuaciones en la evitación de la afectividad negativa que provocan estímulos o situaciones relacionadas con el ámbito escolar, factor I de la School Refusal Assessment Scale-Revised for Children (SRAS-R-C). Para ello, se utilizó también la Child-Adolescent Perfectionism Scale (CAPS) con objeto de evaluar el perfeccionismo. La muestra seleccionada alcanzó un total de 1920 alumnos de entre 15 y 18 años $\left(M_{\text {edad }}=16.31 ; D E=\right.$ 1.00). Los resultados observados muestran diferencias en los grupos Perfeccionismo Socialmente Prescrito (PSP) y en Perfeccionismo Auto Orientado Crítica (PAO-C), con puntuaciones más elevadas en PSP $(d=.19)$ y PAO-C $(d=.42)$ cuanto mayor rechazo escolar. Estos resultados aportan un nuevo conocimiento en el ámbito científico teniendo en cuenta que pocos son los estudios en el país de Ecuador que midan las diferencias entre ambos constructos.

Palabras clave: perfeccionismo socialmente prescrito; perfeccionismo auto orientado crítica; afectividad negativa; rechazo escolar; Ecuador. 


\section{ABSTRACT}

Perfectionism differences depending on low and high and leve lof negative emotion that cause stimuli or situations related to school. Perfectionism is understood as that tendency to establish high performance standards, accompanied by an excessively critical evaluation of them and a great concern for making mistakes, which is why it is related to various pathological problems. In turn, one of the factors of school refusal, evaluates the avoidance of stimuli that provoke negative affectivity, referring to the anxiety caused by the obligation to attend and stay in school. That is, those negative feelings that can cause in some subjects. The objective of the following study was to analyze whether there are statistically significant differences in perfectionism in adolescents with high and low scores in the avoidance of negative affectivity that provoke stimuli or situations related to the school environment, factor I of the School Refusal Assessment Scale Revised for Children (SRAS-RC). For this, the Child-Adolescent Perfectionism Scale (CAPS) was also used to evaluate perfectionism. The selected sample reached a total of 1920 students between 15 and 18 years old $\left(M_{\mathrm{age}}=16.31 ; S D=1.00\right)$. The observed results show differences in the Socially Prescribed Perfectionism (SPP) and Self-Oriented Perfectionism-Critical (SOP-C) groups, with higher scores in SPP $(d=.19)$ and SOP-C $(d=.42)$ the higher school refusal. These results provide new knowledge in the scientific field considering that few studies in the country of Ecuador measure the differences between the two constructs.

Keywords: socially prescribed perfectionism; self-oriented perfectionism-critical; negative affectivity; school refusal; Ecuador.

\section{INTRODUCCIÓN}

El perfeccionismo se conoce como un constructo psicológico con un enfoque multidimensional y multifacético con unas singularidades concretas. Estas características definitorias advierten sobre la elevada habilidad de exigencia que se marca un sujeto en todos sus ámbitos. Es decir, a nivel personal, laboral, social y educativo. En este sentido, si nos basamos en este rasgo de la personalidad, podemos observar que existe un grupo de gente con ciertas características psicológicas que se convierten en desajustes emocionales basados en el desorden y la inadaptación psíquica (Frost, Heimberg, Holt, Mattia y Neubauer, 1993; Stoeber y Otto 2006).

Hamacheck (1978) en sus estudios iniciales halló diferencias existentes entre un perfeccionismo normal y uno neurótico. A este respecto, pudo comprobar cómo el primero de ellos se asociaba con peculiaridades positivas y adaptativas, que promovían aspectos saludables y además eran socialmente aceptadas, dado que el objetivo de estos individuos era la búsqueda de logros y la finalidad de alcanzarlos era vinculante con recompensas personales que aumentaban su autoestima. Sin embargo, por el contrario, según el autor, un perfeccionismo neurótico, se relacionaba con miedo y temor intenso al fracaso. Este pavor, provocaría conductas desadaptativas en tanto en cuanto se instaura un mayor número de dificultades para llegar a lograr el placer y la satisfacción personal.

Obtener la titulación de bachillerato es uno de los objetivos de vida que tienen los adolescentes para poder continuar con sus estudios superiores. No obstante, existe un colectivo, dentro de esta población que rechaza la idea debido a diversas situaciones. Una de ellas, tiene que ver con el rechazo escolar, el cual se entiende como la negativa de un niño o niña a acudir al centro escolar (Kearney y Bates, 2005; Gonzálvez et al., 2016). Es decir, esa conducta negativa que tiene repercusiones desfavorables para la formación integral de los niños y jóvenes en edad escolar, lo que llega a ser contraproducente a nivel social y profesional (Cava y Musitu, 2003). A este respecto, sería interesante medir si el perfeccionismo implica una mayor tasa de rechazo escolar en población juvenil ecuatoriana. 
La práctica del rechazo escolar se acontece de diversas maneras tales como no acudir al aula, o realizarlo de manera esporádica al inicio de las clases hasta que finalmente se decide no acudir más a la institución educativa antes de la edad instaurada (Attwood y Croll, 2006). Evidentemente, en edades tempranas, la importancia o el alcance de poder decidir asistir o no al aula es distinta que en población juvenil. Una de las razones, según Kearney (2005), podría deberse al rechazo de estímulos que provocan la afectividad negativa, referido a la ansiedad que provoca la obligación de asistir y permanecer en la escuela (e.g., ¿Cuántas veces tratas de no ir a la escuela porque si vas te sentirás triste o deprimido?). En este sentido, el autor señala que este perfil de alumnado es reticente a asistir al aula para evitar la presencia de malestares psicosomáticos y estímulos desagradables. De este modo, este tipo de alumnado es capaz de expresar su malestar bajo la manifestación de amenazas por parte de sus iguales, o bajo la declaración de indisposición para poder recibir la educación en su hogar.

Diversos autores afirman de la repercusión negativa sobre la salud mental que tiene la persistencia de rechazo escolar desde edades tempranas hasta la adolescencia si esta no se ataja, desarrollando trastornos de ansiedad (Kearney y Albano, 2004), depresión, problemas de conducta (Egger, Costello y Angold, 2003), soledad y afectivo negativo (Jones y Suveg, 2015) lo que lleva consigo síntomas adyacentes y lamentables efectos sociales y personales. Este dato es preocupante teniendo en cuenta que es una variable con una alta prevalencia en el sistema educativo, si tenemos en cuenta los últimos datos proporcionados por el Ministerio de Educación del Ecuador en la estadística educativa de Quito (Antamba-Chacua, 2015) el cual advierte de cifras donde únicamente el $56.8 \%$ de los jóvenes de zonas urbanas y rurales finalizaron sus estudios de bachillerato.

Evidentemente, son datos que no pueden pasar desapercibidos. En este sentido, es necesario idear mecanismos de solución, que eviten estos conflictos de índole personal que además de acarrear con la vida futura y personal de estos jóvenes, responde negativamente con la prosperidad y la economía del país.

Para ello, el presente estudio tiene como objetivo medir si existen diferencias en altas y bajas puntuaciones de la evitación a la afectividad negativa que provocan los estímulos o situaciones relacionadas con el ámbito escolar, en función de las tres dimensiones de perfeccionismo (Perfeccionismo Socialmente Prescrito [PSP], Perfeccionismo Auto Orientado-Crítica [PAO-C] y Perfeccionismo Auto Orientado-Esfuerzos [PAO-E]) según el estudio de Vicent (2017) en el cual se puso a prueba la Child and Adolescent Perfectionism Scale (CAPS) con población infantil española, y el cual fue replicado por Ortega-Sandoval (2019) en población adolescente ecuatoriana. Es importante, por tanto, entender a qué se deben estos factores. El primero de ellos, PSP, se entiende como el convencimiento de que las personas cercanas y significativas esperan que uno mismo sea perfecto. El PAO-C, se contempla como el perfeccionismo que conlleva a una autocrítica y un miedo a cometer errores. Por último, se llama PAO-E a aquellos deseos y esfuerzos por ser perfectos (Ortega-Sandoval, 2019; Vicent, 2017).

\section{OBJETIVOS DE LA INVESTIGACIÓN}

El objetivo del siguiente estudio es observar si existen diferencias estadísticamente significativas en PSP, PAO-E y PAO-C en función del factor I del SRAS-R-C.

\section{MUESTRA Y/O PARTICIPANTES}

La muestra participante fue seleccionada bajo muestreo aleatorio por conglomerados. Concretamente, la muestra inicial alcanzó un total de 1920 alumnos, con una edad de entre 15 y 18 años, y matriculados en 1ํㅜㄴ 2ำ y $3^{\circ}$ de bachillerato de 3 instituciones públicas de Ecuador. No obstante, 134 participantes fueron excluiros por omisiones en sus respuestas o por no recibir el con- 


\section{DIFERENCIAS DE PERFECCIONISMO EN FUNCIÓN DEL GRADO DE RECHAZO ESCOLAR POR EVITAR LA AFECTIVIDAD NEGATIVA QUE PROVOCAN LOS ESTÍMULOS ESCOLARES}

sentimiento informado. Por lo que, definitivamente, la muestra se compuso de 1786 participantes (Medad $=16.31 ; D E=1.00)$, de los cuales un $51.0 \%$ eran chicos (véase Tabla 1 ).

Tabla 1.

Tabla de contingencia sexo ${ }^{*}$ curso con frecuencias y porcentajes

\begin{tabular}{ccccc}
\hline & \multicolumn{3}{c}{ Curso } & Total \\
\hline Sexo & $1^{\mathrm{o}}$ & $2^{\mathrm{o}}$ & $3^{\mathrm{o}}$ & \\
\hline Chicos & 366 & 308 & 236 & 910 \\
$\%$ & $20.5 \%$ & $17.2 \%$ & $13.2 \%$ & $51.0 \%$ \\
Chicas & 342 & 296 & 238 & 876 \\
$\%$ & $19.1 \%$ & $16.6 \%$ & $13.3 \%$ & $49.0 \%$ \\
Total & 708 & 604 & 474 & 1786 \\
$\%$ & $39.6 \%$ & $33.8 \%$ & $26.5 \%$ & $100.0 \%$ \\
\hline
\end{tabular}

\section{METODOLOGÍA Y/O INSTRUMENTOS UTILIZADOS}

\section{Instrumentos}

School Refusal Assessment Scale-Revised for Children (SRAS-R-C; Kearney, 2002; 2006): La SRAS-R-C es una escala tipo Likert que evalúa el rechazo escolar en población infantojuvenil. El instrumento consta de 24 ítems con 7 tipos de respuesta $(0=$ Nunca; $6=$ Siempre $)$, dividido en cuatro factores subyacentes al rechazo a la escuela: Fl. Evitar la afectividad negativa que provocan los estímulos o situaciones relacionadas con el ámbito escolar (e.g.: ¿Cuántas veces tratas de no ir a la escuela porque si vas te sentirás triste o deprimido?); Fll. Escapar de la aversión social o situaciones de evaluación (e.g.: Si fuera más fácil para ti hacer nuevos amigos, ¿sería más fácil para ti ir a la escuela?); FIII. Búsqueda de la atención de otras personas significativas (e.g.: ¿Cuántas veces preferirías que tus padres te enseñaran en casa en vez de tu profesor/a en la escuela?), y FIV. Búsqueda de refuerzos tangibles fuera del ámbito escolar (e.g.: ¿Cuántas veces rechazas ir a la escuela porque quieres divertirte fuera de la escuela?). Gonzálvez et al. (2016) probaron las propiedades psicométricas de la escala en población española, con niveles de fiabilidad entre .70 y .87.

Child and Adolescent Perfectionism Scale (CAPS; Flett, Hewitt, Boucher, Davidson y Munro, 2000; Flett et al., 2016). En el presente estudio se empleó la versión española de la CAPS Vicent et al. (2017), la cual se trata de un instrumento tipo Likert de 5 puntos ( $1=$ Nada cierto en mí; $5=$ Muy cierto en mí) compuesta por 13 ítems estructurados en 3 dimensiones: FI. PSP (e.g.: Hay personas en mi vida que esperan que yo sea perfecto); FII. PAO-E (e.g.: Intento ser perfecto en todo lo que hago) y FIII. PAO-C (e.g.: Me enfado conmigo mismo cuando cometo un error). Los niveles de fiabilidad para la adaptación española fueron aceptables tanto para el total de la escala $(=.70)$ como para sus tres dimensiones de PSP, PAO-E y PAO-C ( $=.80, .75$ y .72) respectivamente.

\section{Procedimiento}

Se realizó una entrevista con los equipos directivos de las instituciones educativas, con el objetivo de solicitar su colaboración y explicar la finalidad del trabajo de investigación, la temática, la administración y tipo de cuestionarios, así como el respeto a la privacidad.

Asimismo, cabe destacar la importancia del consentimiento informado por escrito de cada uno 
de los representantes legales de los participantes. De ese modo, la administración de cuestionarios fue realizado una vez se obtuvo este consentimiento.

Los participantes fueron informados sobre la finalidad del estudio, su voluntariedad y anonimato. El tiempo de ejecución de los cuestionarios fue de aproximadamente 20 minutos.

\section{Análisis de datos}

Para los análisis de datos llevados a cabo en el estudio se utilizaron pruebas $t$ de Student. Estos análisis fueron realizados con el objetivo de determinar diferencias en las tres dimensiones de perfeccionismo (PSP, PAO-E, y PAO-C) entre población juvenil ecuatoriana en función del primer factor de la SRAS-R-C (Evitar la afectividad negativa que provocan los estímulos o situaciones relacionadas con el ámbito escolar). Se han tenido en cuenta las consideraciones de Cohen (1988) para comparar las puntuaciones, las cuales consideran un pequeño tamaño (.20 - .49), un tamaño moderado $(.50-.79)$ y un tamaño grande $(\leq .80)$.

Asimismo, el reconocimiento de adolescentes con alto y bajo grado de rechazo escolar, fue considerado un nivel alto mediante puntuaciones iguales o superiores al percentil 75 , y un nivel bajo iguales 0 inferiores al percentil 25.

Los análisis estadísticos realizados fueron calculados a través del programa estadístico SPSS 22.0.

\section{RESULTADOS ALCANZADOS}

Los hallazgos observados, según la Tabla 2, muestran diferencias estadísticamente significativas en dos de los factores de perfeccionismo, en función de la evitación de la afectividad negativa que provocan los estímulos o situaciones relacionadas con el ámbito escolar. Las diferencias halladas entre los grupos de adolescentes con altas y bajas puntuaciones del FI de rechazo escolar, resultaron significativas tanto para el PSP, como para el PAO-C. Sin embargo, las diferencias no fueron significativas para el PAO-E.

Tabla 2.

Diferencias en perfeccionismo en adolescentes con altas y bajas puntuaciones en el FI del SRAS-R-C

\begin{tabular}{lllllllllll}
\hline Variable & \multicolumn{2}{c}{$\begin{array}{c}\text { Prueba } \\
\text { Levene }\end{array}$} & \multicolumn{2}{c}{$\begin{array}{c}\text { Altas } \\
\text { Puntuaciones }\end{array}$} & \multicolumn{2}{c}{$\begin{array}{c}\text { Bajas } \\
\text { Puntuaciones }\end{array}$} & \multicolumn{3}{c}{$\begin{array}{c}\text { Significación estadística y } \\
\text { magnitud diferencias }\end{array}$} \\
\hline & $F$ & $p$ & $M$ & $D E$ & $M$ & $D E$ & $t$ & $g . l$. & $p$ & $d$ \\
\hline PSP & 26.53 & $<.001$ & 17.24 & 4.16 & 16.39 & 5.03 & 2.95 & 943.2 & .003 & .19 \\
PAO-E & 8.14 & .004 & 13.56 & 3.41 & 13.34 & 3.89 & .94 & 973.6 & .343 & - \\
PAO-C & 6.15 & .013 & 13.02 & 3.47 & 11.51 & 3.79 & 6.67 & 992.2 & $<.001$ & .42 \\
\hline
\end{tabular}

Nota: FI SRAS = Evitar la afectividad negativa que provocan estímulos o situaciones relacionadas con el ámbito escolar; PSP $=$ Perfeccionismo Socialmente Prescrito; PAO_E = Perfeccionismo Auto Orientado Esfuerzos; PAO_C = Perfeccionismo Auto Orientado Crítica.

Conforme se puede comprobar en la Figura 1, los adolescentes con alto rechazo escolar, concretamente evitación de la afectividad negativa que provocan los estímulos o situaciones relacionadas con el ámbito escolar, obtuvieron mayores puntuaciones que sus compañeros con bajos niveles de rechazo escolar, para el PSP $(d=.19)$ y el PA0-C $(d=.42)$, siendo estas diferencias de pequeña magnitud. 


\section{DIFERENCIAS DE PERFECCIONISMO EN FUNCIÓN DEL GRADO DE RECHAZO ESCOLAR POR EVITAR LA AFECTIVIDAD NEGATIVA QUE PROVOCAN LOS ESTÍMULOS ESCOLARES}

Figura 1. Diagrama de barras para las diferencias en puntuaciones de perfeccionismo en estudiantes con altas y bajas puntuaciones en el Factor I del SRAS.

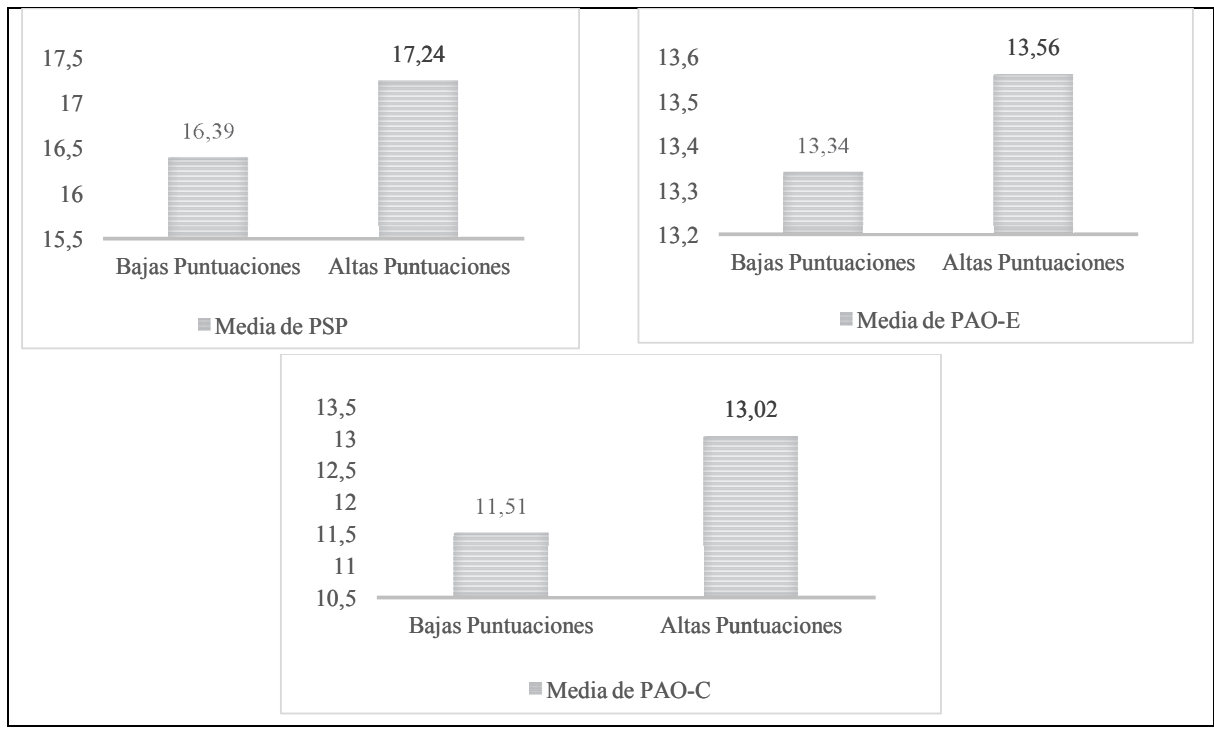

Nota: ${ }^{*}=p<.05 ;{ }^{* *}=p<.01 ;{ }^{* *}=p<.001$.

PSP $=$ Perfeccionismo Socialmente Prescrito; PAO_E = Perfeccionismo Auto Orientado Esfuerzos; PAO_C = Perfeccionismo Auto Orientado Crítica

\section{DISCUSIÓN}

El objetivo del presente estudio fue analizar si existían diferencias significativas del primer factor de la SRAS-R-C, "evitar la afectividad negativa que provocan los estímulos o situaciones relacionadas con el ámbito escolar", tanto en PSP, como en PAO-E y PAO-C, las tres dimensiones de la CAPS.

A partir de los resultados obtenidos se han encontrado diferencias estadísticamente significativas en PSP y PAO-C, con puntuaciones de pequeña magnitud. Sin embargo, el PAO-E no presentó diferencias estadísticamente significativas en rechazo escolar, según el primero de los factores de este constructo.

A este respecto, los hallazgos están en controversia con el estudio realizado por Gonzálvez et al. (2015), el cual no encontró diferencias estadísticamente significativas en el primer factor del SRASR-C y el PSP. No obstante, es posible que la contrariedad de los resultados se deba a diferentes rasgos etarios y culturales, teniendo en cuenta que el estudio de Gonzálvez et al. (2015) fue realizado con niños y niñas residentes en España entre 8 y 12 años ( $M_{\text {edad }}=10.03$; $D E=1.25$ ), y por el contrario este estudio se trata con muestra de adolescentes de Ecuador entre 15 y 18 años de edad $\left(M_{\text {edad }}=16.31 ; D E=1.00\right)$.

Por el contrario, es importante tener en cuenta, según investigaciones previas que los individuos con trastorno de ansiedad social suelen sufrir de un más alto de PSP (Flett y Hewitt, 2014). Esto tiene sentido teniendo en cuenta que el PSP se construye de esas creencias basadas en elevados estándares que son percibidos por parte de los otros. A este respecto, diversos estudios confirman el vínculo entre ansiedad y perfeccionismo (Essau, Conradt, Sasagawa y Ollendick, 2012), incluso en el ámbito educativo (Vicent et al., 2014). 
Por otra parte, por lo que respecta al PAO-C, los hallazgos del presente estudio afirman diferencias estadísticamente significativas en el primer factor del SRAS-R-C, puntuando más alto aqueIlos alumnos con alto rechazo a la escuela por evitar la afectividad negativa que provocan los estímulos o situaciones relacionadas con el ámbito escolar. Es decir, ese miedo, tristeza, nerviosismo, y/o pensamientos negativos que para algunos sujetos les puede causar la escuela. A este respecto, es importante destacar que Vicent (2017) manifestó que aquel alumnado con alto PAO-C, tiende a puntuar más bajo en la apertura a la experiencia, siendo además más inestables emocionalmente que sus compañeros con baja presencia en PAO-C. Asimismo, este estudio demuestra que aquellos alumnos con altos niveles de afectividad negativa tienen mayor probabilidad a presentar alto PAOC. No obstante, por el contrario, Vicent (2017), afirma que aquellos estudiantes con niveles más altos de afectividad positiva tienen mayor probabilidad de presentar un alto PAO-E, así como aqueIlos estudiantes con menores niveles de afectividad negativa suelen presentar mayor probabilidad de un elevado PAO-E. En este sentido, se entiende que el hecho de no observar diferencias significativas de rechazo escolar por evitar la afectividad negativa en PAO-E es debido al vínculo entre la afectividad positiva y esta última dimensión del perfeccionismo.

Cabe tener en cuenta que el trabajo actual tiene algunas limitaciones. Por una parte, sería conveniente ampliar este estudio aplicado a población infantil de Ecuador, para corroborar si los resultados de Gonzálvez et al. (2015) son replicados. Por otra parte, debería replicarse el estudio con población adolescente en población española, por ver si los hallazgos van en consonancia con los del presente estudio.

Asimismo, otra de las limitaciones del estudio versa en torno a las diferencias de sexo y curso. En este sentido, sería interesante medir si existen diferencias estadísticamente significativas según sexo y curso escolar.

\section{CONCLUSIONES}

No obstante, estos resultados sugieren la importancia de seguir avanzando en la investigación científica relacionada con el rechazo escolar y el perfeccionismo en sus diversas dimensiones, teniendo en cuenta todas las asociaciones de carácter negativo que pueden llegar a observarse por estudios previos (Essau et al., 2012; Flett y Hewitt, 2014; Frost et al., 1993; Stoeber y 0tto, 2006; Vicent et al., 2014; Vicent, 2017). A este respecto, se apoya la necesidad de ampliar el conocimiento y diseñar tratamientos de intervención y/o prevención ante diversos casos de rechazo escolar, considerando entre ellos la evitación de la afectividad negativa que provocan los estímulos o situaciones relacionadas con el ámbito escolar y su influencia de PSP y PAO-C sobre la negativa a acudir al centro escolar.

\section{REFERENCIAS BIBLIOGRÁFICAS}

Antamba-Chacua, L. (2015). Estadística Educativa, Reporte de indicadores, 1 (1), 1-30.

Attwood, G. y Croll, P. (2006). Truancy in secondary school pupils: Trajectories and pupil perspectives. Research Papers in Education, 21 (4), 467-484. doi: 10.1080/02671520600942446

Cava, M. J. y Musitu, G. (2003). Dificultades de integración social en el aula: relación con la autoestima y propuestas de intervención. Informació psicológica, 83, 60-68.

Cohen, J. (1988). Statistical power analysis for the behavioral sciences. Hillsdale, NJ: Erlbaum.

Egger, H. L., Costello, J. E. y Angold, A. (2003). School refusal and psychiatric disorders: a community study. Journal of the American Academy of Child \& Adolescent Psychiatry, 42 (7), 797807, 797-807. doi: 10.1097/01.CHI.0000046865.56865.79 
Essau, C.A., Conradt, J., Sasagawa, S. y Ollendick, T. H. (2012). Prevention of anxiety symptoms in children: results from a universal school-based trial. Behavior Therapy, 43, 450-464. doi: 10.1016/j.beth.2011.08.003

Flett, G. L. y Hewitt, P. L. (2014). Perfectionism and perfectionistic self-presentation in social anxiety. In S. G. Hofman, y P. M. Dibartolo (Eds.), Sociall anxiety: Clinical, developmental, and social perspectives (pp.160-183) (3rd ed). London, UK: Elservier

Flett, G. L., Hewitt, P. L., Boucher, D. J., Davidson, L. A. y Munro, Y. (2000). The Child-Adolescent Perfectionism Scale: development, validation, and association with adjustment. Manuscrito inédito, York University, Toronto, Ontario, Canadá.

Flett, G. L., Hewitt, P. L., Besser, A., Su. C., Vaillancourt, T., Boucher, D., ... Gale, O. (2016). The child-adolescent perfectionism scale: development, psychometric of Psychoeducational Assessment, 34 (7), 634-652. doi: 10.1177/07342829166651381

Frost, R. O., Heimberg, R. G., Holt, C. S., Mattia, J. I. y Neubauer, A. L. (1993). A comparison of two measures of perfectionism. Personality and Individual Differences, 14 (1), 119-126. doi: 10.1016/0191-8869(93)90181-2

Gonzálvez, C., Inglés, C. J., Kearney, C. A., Vicent, M., Sanmartín, R. y García-Fernández, J. M. (2016). School Refusal Assessment Scale-Revised: Factorial Invariance and Latent Means Differences across Gender and Age in Spanish Children. Frontiers in Psychology, 7, 1-10. doi: 10.3389/fpsyg.2016.02011

Gonzálvez, C., Vicent, M., Inglés, C. J., San Martín, N. L., García-Fernández, J. M. y MartínezMonteagudo, M. C. (2015). Diferencias en Perfeccionismo Socialmente Prescrito en función del rechazo escolar. International Journal of Developmental and Educational Psychology, 1 (1), 455461. doi: 10.1760/ijodaep.2015.n1.v1.47

Hamacheck, D. (1978). Psycho-dynamics of normal and neurotic perfectionism. Phychology. 15, 27-33

Jones, A. M. y Suveg, C. (2015). Flying under the radar: School reluctante in anxious youth. School Mental Health: A multidisciplinary Research and Practice Journal, 7 (3), 212-223. doi: 10.1007/s12310-015-9148-x

Kearney, C. A. (2002). Identifiyng the Function of School Refusal Behavior: A Revision of the School Refusal Assessment Scale. Journal of Psychopathology and Behavioral Assessment, 24 (4) 235245.

Kearney, C. A. (2005). Confirmatory Factor Analysis of the School Refusal Assessment ScaleRevised: Child and Parent Versions. Journal of Psychopathology and Behavioral Assessment, 28 (3), 139-144. doi: 10.1007/s10862-005-90005-6

Kearney, C. A. (2006). Confirmatory factor analysis of the School Refusal Assessment Scale-Revised: Child and parent versions. Journal of Psychopathology and Behavioral Assessment, 28 (3), 139-144. doi: 10.1007/s10862-005-9005-6

Kearney, C. A. y Albano, A. M. (2004). The functional profiles of school refusal behavior: Diagnostic aspects. Behavior Modification, 28(1), 147-161. doi: 10.1177/0145445503259263

Kearney, C. A. y Bates, M. (2005). Addressing school refusal behavior: suggestions for frontline professionals. Children and Schools, 27 (4), 207-216. doi: 10.1093/cs/27.4.207

Ortega- Sandoval, V. N. (2019). Rechazo escolar del alumnado de Bachillerato en la ciudad de Quito (Tesis doctoral). Universidad de Alicante, Alicante, España.

Stoeber, J. y Otto, K. (2006). Positive conceptions of perfectionism: Approaches, evidence, challengs. Personality and Social Psychology review, 10 (4), 295-319. doi: 10.1207/s15327957pspr1004_2 


\section{PSICOLOGIA, INFANCIA Y EDUCACIÓN}

Vicent, M. (2017). Estudio del perfeccionismo y su relación con variables psicoeducativas en la infancia tardía (Tesis doctoral). Universidad de Alicante, Alicante, España.

Vicent, M., Gonzálvez, C., Lagos-San Martín, N., Gomis, N., Inglés, C. J. y García-Fernández, J. M. (2014). El perfeccionismo como predictor del rechazo escolar. En Espada, Orgilés, Piqueras y Carratalá (comps.), IV Symposium nacional de psicología clínica y de la salud con niños y adolescentes (pp.73). Madrid: Pirámide 
